\title{
Therapeutic approach for osteoporotic animal model by bone marrow mesenchymal stem cells
}

\author{
Abdullah S. M. Aljohani ${ }^{1 *}$, Abdel Kader A. Zaki ${ }^{1}$, Waleed Al Abdulmonem², Zafar Rasheed ${ }^{3}$, Fahad A. Alhumaydhi ${ }^{4}$, \\ Faten A. M. Abo-Aziza ${ }^{5}$ \\ ${ }^{1}$ Department of Veterinary Medicine, College of Agriculture and Veterinary Medicine, Qassim University, Buraydah, Saudi Arabia. \\ ${ }^{2}$ Department of Pathology, College of Medicine, Qassim University, Buraydah, Saudi Arabia. \\ ${ }^{3}$ Department of Medical Biochemistry, College of Medicine, Qassim University, Buraydah, Saudi Arabia. \\ ${ }^{4}$ Department of Medical Labratories, College of Applied Medical Sciences, Qassim University, Buraydah, Saudi Arabia. \\ ${ }^{5}$ Department of Parasitology and Animal Diseases, Veterinary Research Institute, National Research Centre, Cairo, Egypt.
}

\begin{tabular}{l}
\hline ARTICLE INFO \\
\hline Received on: $15 / 06 / 2021$ \\
Accepted on: 09/10/2021 \\
Available Online: 05/01/2022 \\
\hline Key words: \\
Bone biomarkers, bone \\
marrow, mesenchymal stem \\
cell, osteoporosis, rat model.
\end{tabular}

\section{INTRODUCTION}

Osteoporosis (OP) is a systemic metabolic bone disease manifested by poor mineral density and deteriorated skeleton, which is common in every population all over the globe (Drake et al., 2015). Numerous previously established theories contributed to its development but the most common was found to be a disturbance

\footnotetext{
*Corresponding Author

Abdullah S. M. Aljohani, Department of Veterinary Medicine, College of Agriculture and Veterinary Medicine, Qassim University, Buraydah, Saudi Arabia.E-mail:jhny@qu.edu.sa
}

\begin{abstract}
Osteoporosis (OP) is a common debilitating metabolic bone disorder manifested by poor mineral density and deteriorated skeleton. Stem cell therapies are being considered as alternative therapeutic modalities. This study determined the therapeutic potential of mesenchymal stem cell (MSC) application in osteoporotic rats. To achieve this, an OP animal model was developed through ovariectomy and glucocorticoids in rats. MSCs were isolated from the same breed of rats and cultured MSCs were administered to osteoporotic rats via vein injections. After 4 and 8 of MSCs administration, rats were sacrificed and all desired components, including serum and joints, were isolated. The present findings showed that the transplantation of MSCs into osteoporotic rats enhanced osteoblastic differentiation and function reverting OP. Calcium deposition, bone-specific alkaline phosphatase activity, and serum osteocalcin concentration were significantly elevated in MSCs-treated osteoporotic rats $(p<0.05)$. Notably, their levels were much higher in osteoporotic rats treated for 8 weeks than in those treated for 4 weeks $(p<0.05)$. Moreover, the histological image of the femur head showed uniform thinning of trabeculae (TB) in osteoporotic rats, resulting in the widening of intertrabecular spaces and disjointed bony ossicles interrupted by virtue of expanded fatty bone marrow spaces. Interestingly, after MSCs treatment, cancellous bone TB appeared normal with red bone marrow. Furthermore, a photomicrograph of osteogenic differentiation of MSCs of MSCs-treated osteoporotic rats showed significantly high scattered red calcified nodules $(p<0.05)$. In conclusion, this study illustrates that the transplantation of MSCs into osteoporotic rats provides a supplement for bone architecture and enhances osteoblastic differentiation and function, thus reverting OP. This work presents a unique method as a future application for the treatment of human OP.
\end{abstract}

in the balance of bone formation and resorption, which may lead to its onset due to the excessive loss of bone tissue (Thomsen et al., 2012). Moreover, dysfunction of a number of hormones and interleukins was also found to have a link with the onset of OP (Chen et al., 2016). Estrogen deficiency due to menopause was reported to activate osteoclasts, resulting in a reduction in bone formation (Miyamoto, 2015). At present, the drug treatment for primary OP targets symptoms and complications, without bone targeting of bone formation (Cosman et al., 2014). The therapeutic mechanism for parathormone treatment includes the acceleration of bone formation but subsequently stimulates bone resorption (Makras et al., 2015). Correcting abnormal bone revolution may \\ (https://creativecommons.org/licenses/by/4.0/).}


improve bone weight and reduce the chance of fractures. However, pharmacotherapy can be prohibitive in cost, take a long time to be effective, and lead to adverse reactions. Bisphosphonate therapy may lead to extensive suppression of bone development and even necrosis of the mandibular bone (Rousselle and Heymann, 2002). The nonpharmacologic low-intensity pulsed ultrasound and physical exercise trials were done but yet it needs more justification according to the variability of sex, age, and bone status (Sun et al., 2020). Additionally, some of these therapies employ virus-mediated gene or protein delivery, posing potential biological risks to the patient (Chen et al., 2002). Therefore, it is essential to develop improved therapies for OP.

Recently, mesenchymal stem cell (MSC)-based therapies have been implemented for different diseased animal models and some of them are under consideration, but their success in clinical applications is yet to be established (Chen et al., 2002). Studies have also shown that MSCs can produce a number of cellular components that are related to bone regeneration but their effect on the curve of bone formation and their applicability in therapeutics against the onset of OP has not yet been clearly defined. Moreover, live-cell industrialization and preservation have also not yet been available for clinical application. Furthermore, cell therapy alone also failed due to its short shelf life (Littman and Abo, 2015). Low bone formation was observed in osteoporotic conditions due to scanty recruitment and invasion of MSCs to the defect bone for bone healing (Almeida et al., 2019; Sanghani-Kerai et al., 2017). But still, stem cells possess many unique features such as selfrenewal ability, potential of multiple cell lineages differentiation, and potential in regeneration of tissues (Fortier and Travis, 2011).

This study determined the therapeutic potential of MSCs application in osteoporotic rats. In light of the works discussed, the present study hypothesized that the transplantation of MSCs into osteoporotic rats may augment bone architecture and enhance osteoblastic differentiation and function, which might result in the reversion of OP conditions. To test this hypothesis, an OP animal model was developed by ovariectomy and glucocorticoids in rats. MSCs were isolated from the same breed of rats and cultured MSCs were administered to osteoporotic rats via vein injections. After 4 and 8 weeks of MSCs administration, rats were sacrificed, all desired components including serum and joint were isolated, and the levels of all top biomarkers involved in OP were determined. The present data proved that the transplantation of MSCs into osteoporotic rats provides a supplement for bone architecture and enhances osteoblastic differentiation or functions, thus reversing the key features of OP.

\section{METHODS}

\section{Ethical approval}

This work was conducted under the international ethical guidelines for animal research and was permitted by the Animal Research Ethical Committee of Qassim University (Approval No. 3739-cavm-2018-1-14-S).

\section{Development of osteoporotic animal model}

Female Wistar rats ( 50 days old, weighed 150-170 g) were used for induction of OP by ovariectomy and glucocorticoids. The rats were housed in equal groups, split into five rooms, fed standard ration, and given water ad libitum. For ovariectomy, a bilateral operation was performed with a dorsal approach and all steps were conducted under ether anesthesia to minimize animal suffering (Wronski et al., 1985). The incision was sealed with interrupted sutures using a nylon 4.0. Sham control rats were exposed to sham surgery in which ovaries were exteriorized. Two days after ovariectomy, administration of dexamethasone phosphate $(1 \mathrm{mg} / \mathrm{kg}$ bwt) by intraperitoneal (i.p.) injections was performed daily for a period of 8 weeks, or with saline as untouched control. For ensuring OP, 10 animals were randomly selected; blood samples were collected for estradiol analysis (E2) using an enzyme-linked immunosorbent assay (ELISA) kit (Cayman Chemical, MI, USA). Measurements were performed every 2 weeks for 8 weeks after ovariectomy until serum E2 (>50\%) was significantly detected compared with the sham control group.

\section{Isolation of MSCs from bone marrow}

Five female Wistar albino rats 50 days old were used for isolation of MSCs from bone marrow as described previously with some modification (Abo-Aziza et al., 2019a). Briefly, sodium pentobarbital anesthesia was used for euthanization of rats and bone marrow was harvested from hind limbs. The limbs were cut over the hip and under the ankle, keeping the bone ends to ensure bone marrow sterility. Bones were washed three times in a dish using phosphate buffer saline (PBS). The ends of each bone were snipped off to extract more bone marrow in sterile PBS. A $10 \mathrm{cc}$ syringe containing prewarmed complete conditioned Dulbecco's modified Eagle's media [1.0 g/l glucose + fetal bovine serum (FBS) $10 \%$, Sigma-Aldrich, USA] was used and the media were pushed several times by the needle through the bone shaft to extract all the red marrow into a $100 \mathrm{~mm}$ Petri dish containing $1 \mathrm{ml}$ heparin $(2,000 \mathrm{IU} / 0.2 \mathrm{ml})$. The cell mixture was pipetted up and down several times using a syringe. The suspension was washed twice by centrifugation using PBS for tissue remnants removal. Alpha minimum essential medium ( $\alpha$-MEM), (Lonza, Germany) was then added, and the bone marrow was used on the equal volume of buffer solution of sodium carbonate buffer solution $(0.1 \%)$ for lyses of erythrocytes, as described previously (Lee et al., 2015). The mononuclear cells (MNC) were separated and were grown as described previously (Abo-Aziza et al., 2019b; Lee et al., 2015).

\section{Immunophenotype analysis for isolated MSCs}

The immunophenotype analysis for bone marrow MSCs was performed through the detection of cell surface markers using the flow cytometry as described previously (Ssadh et al., 2019). All major cell surface markers for MSCs such as CD34, CD45, CD14, CD105, CD166, CD90, CD73, CD146, and CD106 were assessed using FACS Calibur flow cytometer (BD Bioscience, San Jose, CA).

\section{Application of stem cells to osteoporotic rats}

Stem cell therapy was applied to develop osteoporotic rats. MSCs ( 0.5 million/rat) were delivered via tail vein injection with two different time points of harvest, 4 and 8 weeks after stem cell injection. Rats were divided into four groups with $n$ of 20 per each group as follows: Group 1: untouched control; Group 2: sham control; Group 3: $\alpha$-MEM (OP +ve control); Group 4: OP + MSCs. Over half of the animals of the last two groups were sacrificed after 4 weeks via overdose i.p. injection of $3 \%$ sodium pentobarbitone 
(50 mg/kg), followed by the collection of blood and bone samples, while the other half were sacrificed 8 weeks after injection.

\section{Biomarker evaluation: serum osteocalcin (OCN), serum bone-specific alkaline phosphatase (B-ALP), calcium levels, and phosphorus levels}

The experimental rats were sacrificed by the use of sodium pentobarbitone, following which desired components, such as blood, joint tissues, bone tissue, and bone marrow, were collected for the biomarkers analysis to detect the stem cell application on osteoporotic rats. Serum samples obtained from the experimental rats were evaluated for OCN levels by eBioscience ELISA kits (San Diego, CA). B-ALP was quantified by Metra Biosystems ELISA kits (Quidel Corp., CA, USA). Meanwhile, the levels of calcium $(\mathrm{Ca})$ and phosphorus $(\mathrm{P})$ were measured by colorimetric kits (Sigma-Aldrich, USA).

\section{Stem cell sampling}

Bones were also collected from the experimental rat's groups and the acquired bone marrow was harvested to isolate and culture MSCs for osteoblastic differentiation. MSCs (passage 3) were tested for viability by trypan blue stain and 3-(4,5-dimethylthiazol2-yl)-2,5-diphenyltetrazolium bromide (MTT), (Sigma-Aldrich, USA) assays before culturing in osteogenic media as described previously (Alabdulmonem et al., 2018; Rasheed et al., 2010). The cell suspension was done at an appropriate concentration of $1 \times 10^{5} / \mathrm{ml}$ in $\alpha$-MEM as described previously (Quan et al., 2016). The proliferation capability of the stem cells was measured in both primary cultures and subcultures. Cells were incubated with 0.2 $\mathrm{mg} \mathrm{MTT} / \mathrm{ml} \alpha$-MEM media for 1 hour at $37^{\circ} \mathrm{C}$ in 6 -well plates to form formazan. The media were then collected with the addition of $1 \mathrm{ml}$ isopropanol containing $0.04 \mathrm{~N} \mathrm{HCl}$. Formazan quantity was assayed calorimetrically at a wavelength of $570 \mathrm{~nm}$ after 5 minutes of shaking (Zaki et al., 2020).

\section{Osteoblastic differentiation}

Osteoblastic differentiation was used as described previously (Abo-Aziza et al., 2019a). Briefly, the culture medium of passage 3 confluent cultures was replaced by an osteogenic medium containing $\alpha$-MEM with $10 \%$ FBS, penicillin-streptomycin, and GlutaMAX complemented with $100 \mu \mathrm{M}$ L-ascorbic acid 2-phosphate, $10 \mathrm{nM}$ dexamethasone, $2 \mathrm{mM}$ L-glutamine, and $2 \mathrm{mM} \beta$-glycerophosphate (Lonza, Germany). The differentiation of cultures continued for 3 weeks. The media were changed every 2 days. Calcified tissues and mineralization potentiality inside differentiated cells were evaluated using Alizarin red stain, as described previously (Huang et al., 2009).

\section{$B$-ALP activity for osteogenic differentiation}

B-ALP activity was assayed to indicate osteogenic differentiation as described previously (Choi et al., 2005) and the levels of its activity were measured using the B-ALP-specific assay kit (Abcam, Cambridge, UK).

\section{Calcium deposition assay for osteogenic differentiation}

The differentiation of MSCs to bone tissue was indicated by the amount of calcium deposited (Salasznyk et al., 2004). A fixed number of colonies from $80 \%$ confluent plates were washed twice with PBS. Calcium was extracted from cells by agitation for five hours at $4^{\circ} \mathrm{C}$ and centrifugation at low speed using hydrochloric acid extracting solution $(0.5 \mathrm{~N})$. Ca was assessed in the supernatant by colorimetric assay kit (Abcam, Cambridge, UK).

\section{Bone histomorphometry}

The femurs of all rats were separated from their attached muscles and fixed immediately for 2 days in neutral buffered formaldehyde (10\%, Sigma-Aldrich, USA). After that, the distal femurs of the trabecular bone were collected from the midline longitudinal sections and mediolateral plane and decalcified for 8 days with the decalcifying solution, which consisted of formic acid $24.4 \%$ and sodium hydroxide $0.5 \mathrm{~N}$ (Sigma-Aldrich, USA). Paraffin blocks were possessed, and $5 \mathrm{~mm}$ thick sections were serially performed and stained with hematoxylin and eosin stain (H\&E) and examined, as previously described by Eça et al. (2009).

\section{Statistical analysis}

The data found through the results were expressed as mean \pm standard error (SE). Statistical Package for the Social Sciences version 20.0 software was used. Analysis of variance was done for all groups in the study to find out if the results were significant. Ca deposition, phosphorous, B-ALP activity, and OCN concentrations in serum as well as $\mathrm{Ca}$ and B-ALP concentrations in lysed differentiated MSCs were compared through the Student $t$-test, and $p$-values $<0.05$ and $<0.01$ were considered as statistically significant. The viability $\%$ was calculated by the following formula: $100^{*}$ number of viable cells/number of harvested cells.

\section{RESULTS}

\section{MSCs morphology}

MNCs were isolated from rat bone marrow and seeded to adhere to the culture dish bottom. One to two days later, cells were monitored using an inverted microscope. It was found that the cells started to develop extensions and some cells appeared spindled as fibroblasts. The proliferation and propagation of cells continued from $50 \%$ confluence (Fig. 1A) until the targeted $80 \%$ confluence was achieved, where the dish bottom appeared covered with cells after 3 weeks (Fig. 1B). Throughout the expansion cultures in $\mathrm{P} 2$ and $\mathrm{P} 3$, the cell number continued to propagate. The subcultures from the $80 \%$ confluence cells have a tendency to grow faster, so the cultures take a shorter time to reach confluence as compared to primary cultures.

\section{Phenotypic analysis}

Immunophenotypic analysis for characterization of MSCs surface markers was performed using the FACS Calibur machine (Fig. 1). The positive stained population indicated the expression of MSCs surface markers. The results showed a low +ve stained population of MSCs for CD34 (10\%), CD45 (4.9\%), and CD14 (26\%) that indicated negative expression. However, a high +ve stained population was recorded for CD105 $(73.9 \%)$, CD166 (23\%), CD90 (46.6\%), CD73 (50.1\%), CD146 (58.6\%), and CD106 (62.9\%) indicating positive expression. The $-\mathrm{ve}$ sample with the untreated isotope was used as the control. It expressed a very low positive population $(0.6 \%)$. 

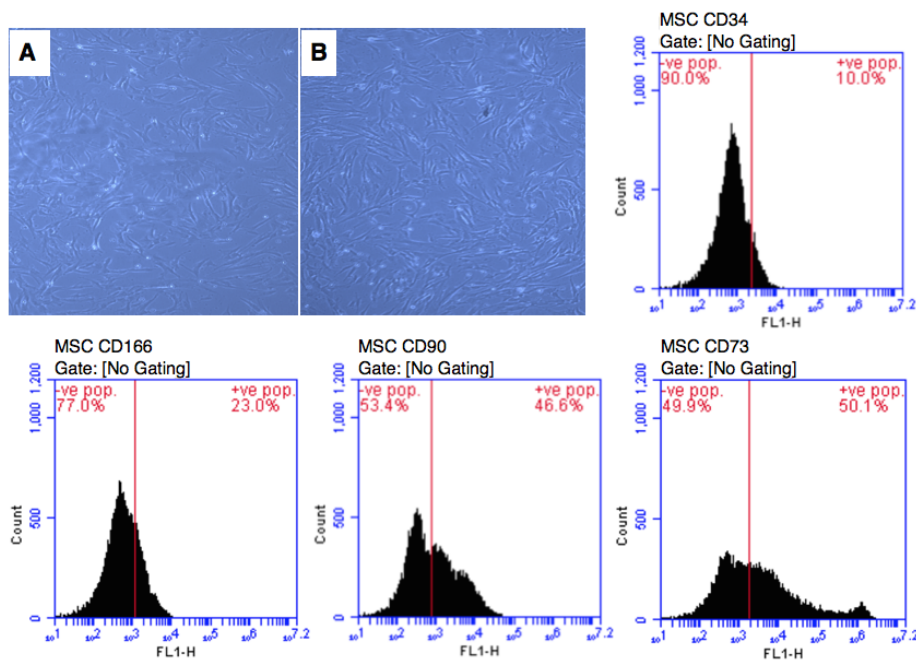

MSC CD90
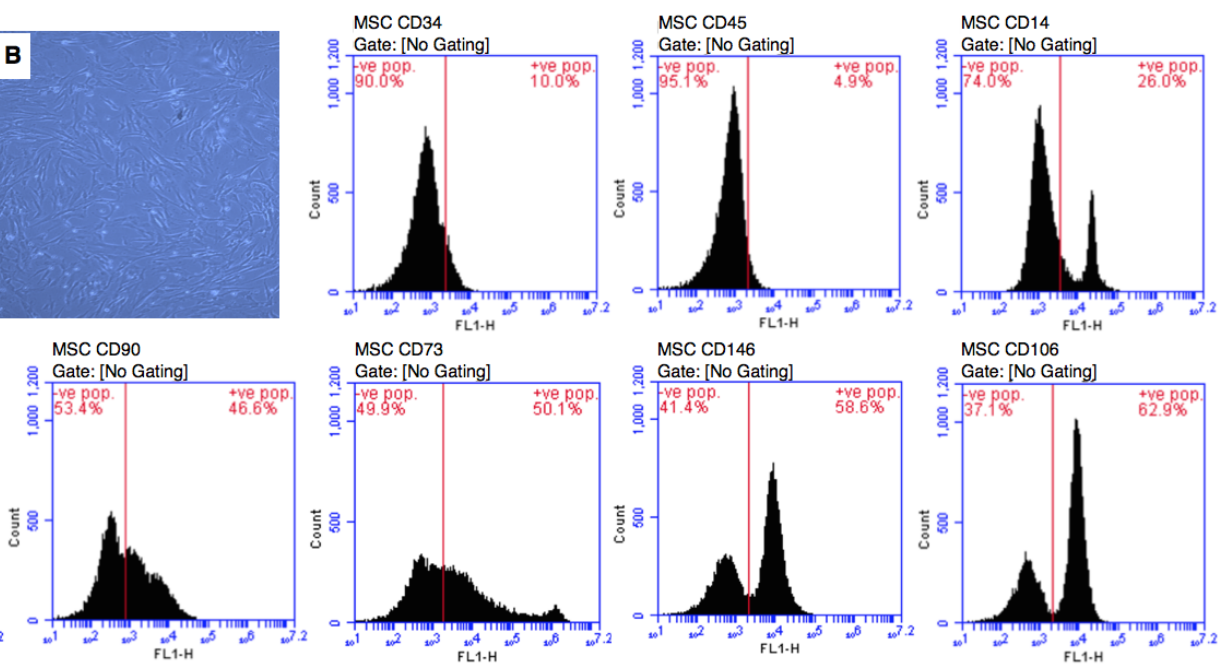

MSC CD146
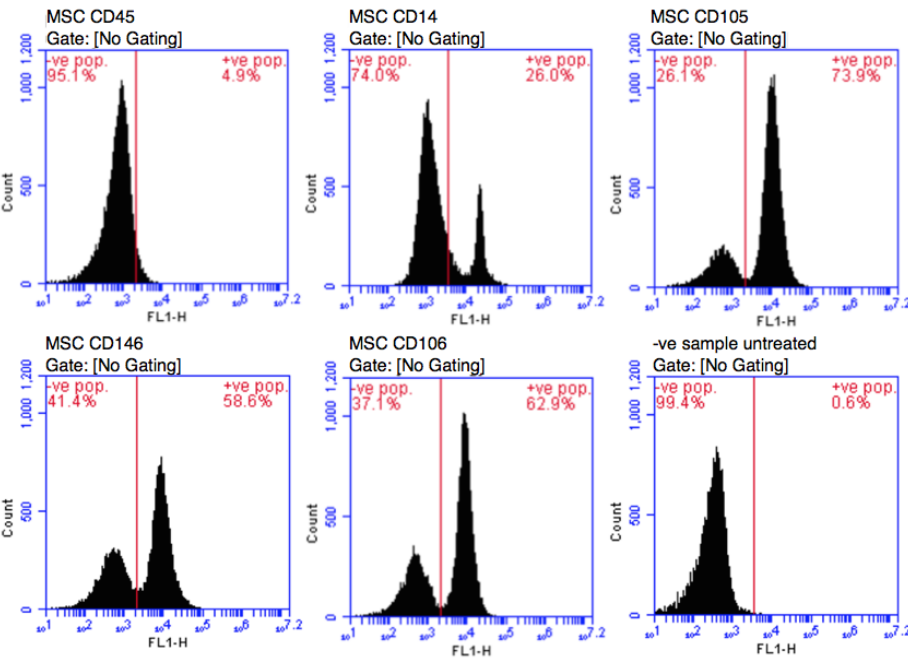

-ve sample untreated

Oิ Gate: [No Gating]

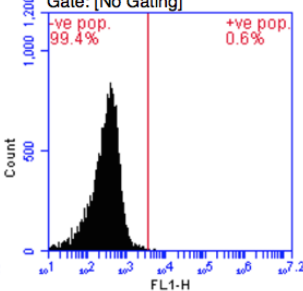

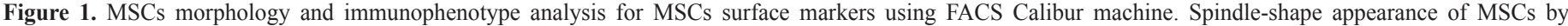

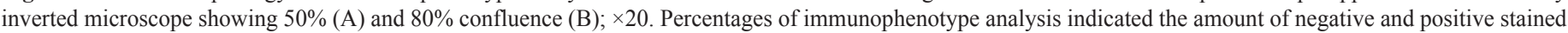

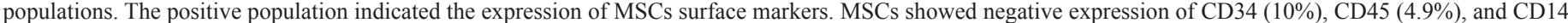

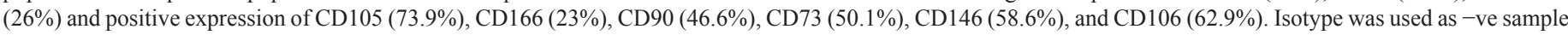
untreated control $(0.6 \%)$. MSCs: mesenchymal stem cells, Pop: population.
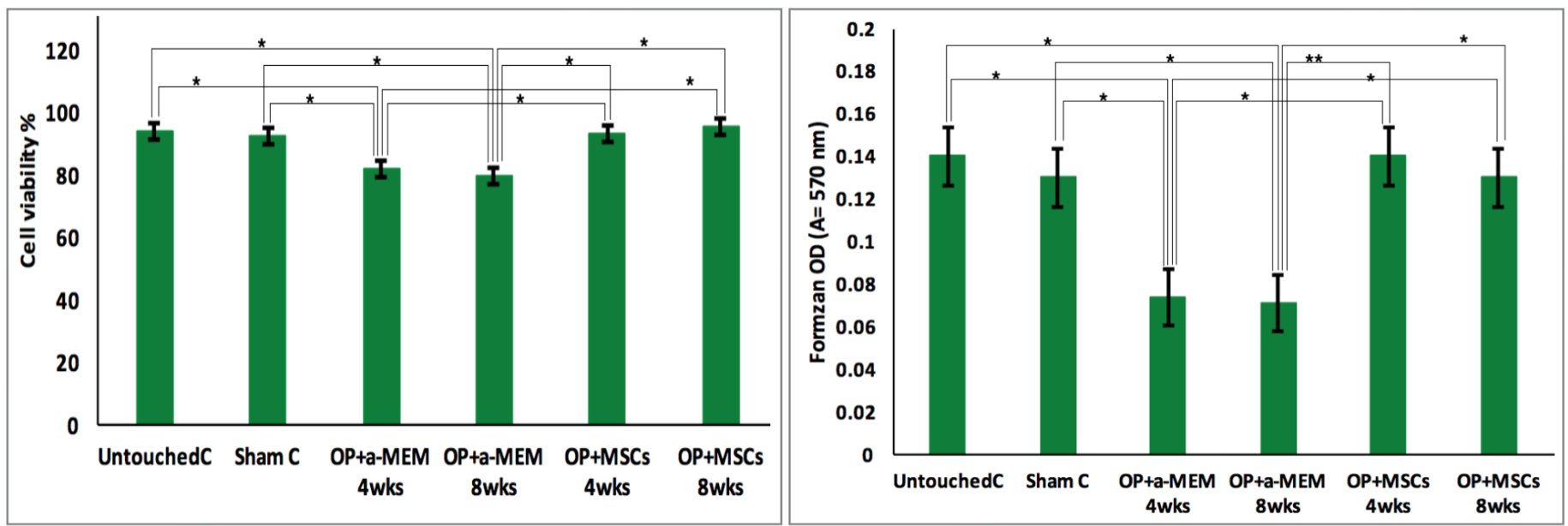

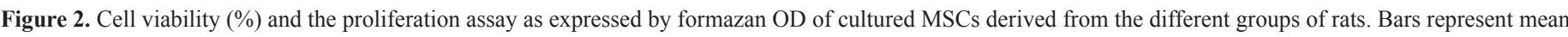

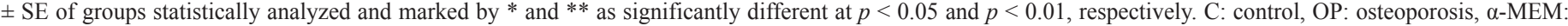
alpha-modified Eagle medium, and MSCs: mesenchymal stem cells.

\section{Cell viability and MTT assay}

Cell viability percentage and the proliferation of cultured MSCs derived from the different groups of rats were measured (Fig. 2). The cell viability percentage of sham-operated rats was $92.52 \%$, which was not significantly different from the untouched control group (94.13\%). However, the cell viability percentage of MSCs derived from OP at $4(82.27 \%)$ and $8(79.83 \%)$ weeks experienced a significant decline compared to the untouched control and sham control groups $(p<0.05)$. It was noticed that cell viability of MSCs derived from OP + MSCs after $4(93.52 \%)$ and 8 (95.36\%) weeks after treatment showed significant elevation compared to OP at 4 and 8 weeks $(p<0.05)$. The quantity of formazan was measured using an MTT assay to evaluate the proliferation of MSCs (Fig. 2). The results showed that the formazan optical densities (ODs) of MSCs of sham-operated and untouched control rats were $0.14 \pm$ 0.04 and $0.13 \pm 0.02$, respectively, and did not differ significantly. It was observed that formazan ODs of MSCs derived from OP at either 4 weeks $(0.074 \pm 0.003)$ and 8 weeks $(0.071 \pm 0.004)$ were significantly declined compared to the untouched control and sham control groups $(p<0.05)$. However, formazan ODs of MSCs derived from OP + MSCs at 4 weeks $(0.14 \pm 0.01)$ and 8 weeks $(0.13 \pm$ $0.006)$ after treatment were significantly elevated compared to $\mathrm{OP}$ at the fourth and eighth weeks ( $p<0.05$ and $p<0.01$, respectively).

\section{Serum biomarkers levels of MSCs-treated and MSCs- nontreated osteoporotic rats}

Serum biomarkers OCN, calcium, phosphorus, and B-ALP levels were determined after OP induction as well as after 

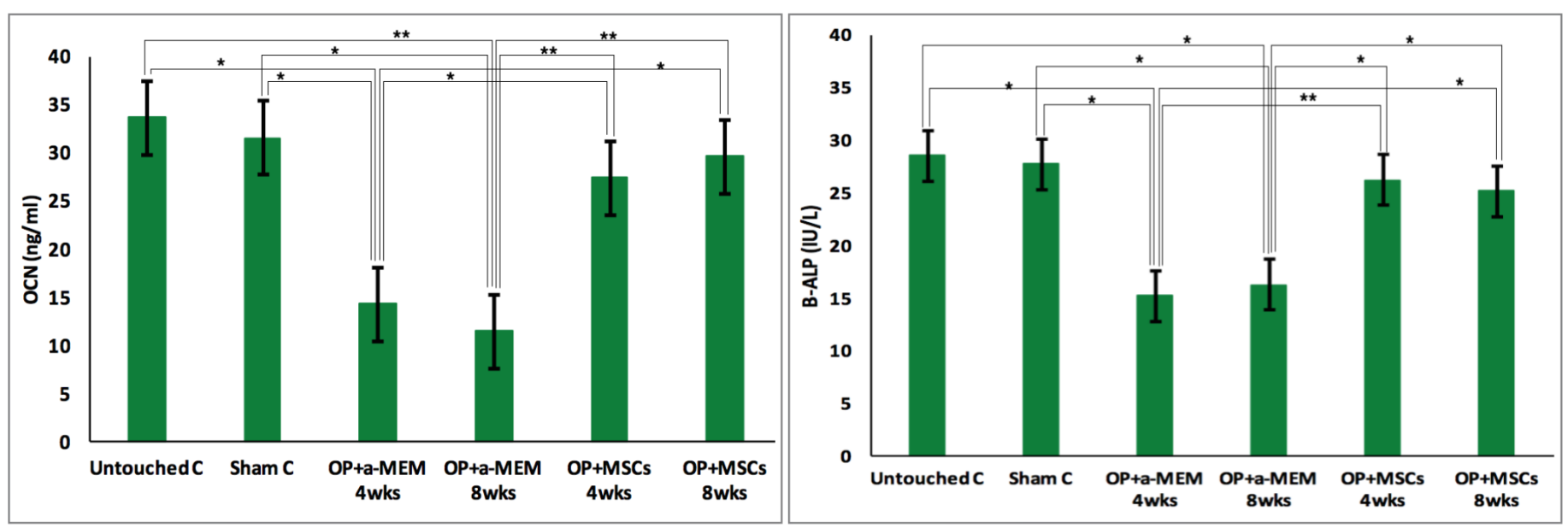

Figure 3. OCN concentration and B-ALP activity in the serum of the different groups of rats. Bars represent mean \pm SE of groups and marked by * and ** as significantly different at $p<0.05$ and $p<0.01$, respectively. C: control, OP: osteoporosis, $\alpha$-MEM: alpha-modified Eagle medium, and MSCs: mesenchymal stem cells.

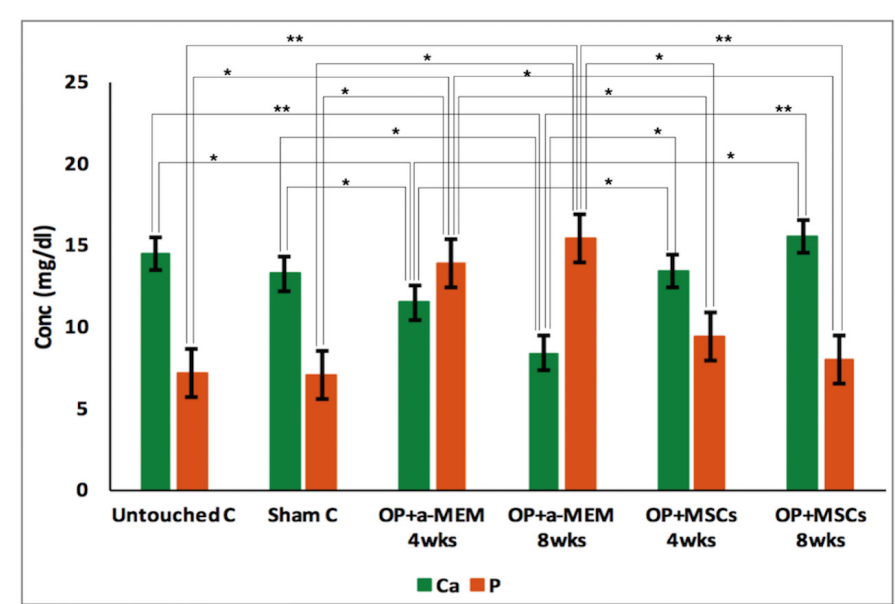

Figure 4. Calcium and phosphorous concentrations in the serum of the different groups of rats. Bars represent mean $\pm \mathrm{SE}$ of groups and marked by $*$ and $* *$ as significantly different at $p<0.05$ and $p<0.01$, respectively. C: control, OP: osteoporosis, $\alpha$-MEM: alpha-modified Eagle medium, and MSCs: mesenchymal stem cells.

MSCs treatment after 4 and 8 weeks and are shown in Figures 3 and 4. OCN concentrations were measured in the different groups by ELISA kits. A significant decline was observed in the serum concentrations of OCN of OP at 4 weeks $(14.29 \pm 1.53 \mathrm{ng} / \mathrm{ml})$ and 8 weeks $(11.51 \pm 1.09 \mathrm{ng} / \mathrm{ml})$ as opposed to the untouched control $(33.62 \pm 4.23 \mathrm{ng} / \mathrm{ml})$ and sham control $(31.48 \pm 2.88 \mathrm{ng} / \mathrm{ml})$ groups $(p<0.01)$. After treatment with MSCs, the results revealed a significant elevation in OCN concentrations in the sera of treated rats in the OP + MSCs group at 4 weeks $(27.39 \pm 2.87 \mathrm{ng} / \mathrm{ml})$ and 8 weeks $(29.61 \pm 3.11 \mathrm{ng} / \mathrm{ml})$ compared to OP at 4 weeks and 8 weeks ( $p<0.05$ and $p<0.01$, respectively).

B-ALP activity was assayed in the serum of different groups of rats (Fig. 3). A significantly decreased serum B-ALP activity was noticed in OP at 4 weeks and 8 weeks $(15.17 \pm 1.13$ and $16.23 \pm 2.65 \mathrm{IU} / 1$, respectively) compared to the untouched control $(28.49 \pm 3.11 \mathrm{IU} / 1)$ and sham control $(27.68 \pm 3.08 \mathrm{IU} / 1)$ groups $(p<0.01$ and $p<0.05$, respectively). However, a significant elevation was observed in B-ALP activity in the serum of the OP + MSCs group after 4 weeks $(25.11 \pm 3.15 \mathrm{IU} / 1)$ and 8 weeks $(26.22 \pm 3.87 \mathrm{IU} / \mathrm{l})$ compared to OP $(p<0.01)$. Sham-operated rats did not show any significant differences in all serum measured parameters compared to untouched control.

Calcium and phosphorus concentrations were measured in the serum of rats using the calorimetric method (Fig. 4). Serum Ca concentration was significantly lower in OP at 4 weeks $(11.45$ $\pm 1.07 \mathrm{mg} / \mathrm{dl})$ and 8 weeks $(8.36 \pm 1.66 \mathrm{mg} / \mathrm{dl})$ after induction compared to the untouched control $(14.42 \pm 1.11 \mathrm{mg} / \mathrm{dl})$ and sham control $(13.23 \pm 1.23 \mathrm{mg} / \mathrm{dl})$ groups $(p<0.05)$. It was observed that serum $\mathrm{Ca}$ concentrations were significantly elevated after MSCs treatment as shown in the OP + MSCs group after both 4 weeks $(13.39 \pm 1.34 \mathrm{mg} / \mathrm{dl})$ and 8 weeks $(15.50 \pm 1.67 \mathrm{mg} /$ dl) compared to OP $(p<0.05)$. Serum phosphorus concentration was significantly elevated $(p<0.05)$ in OP at 4 and 8 weeks $(13.89 \pm 1.43$ and $15.36 \pm 1.98 \mathrm{mg} / \mathrm{dl}$, respectively) compared to the untouched control $(7.11 \pm 0.63 \mathrm{mg} / \mathrm{dl})$ and sham control $(6.99 \pm 0.98)$ groups. However, OP + MSCs showed a significant decrease in the concentration of serum $\mathrm{P}$ after 4 and 8 weeks of MSCs treatment $(9.41 \pm 1.01$ and $7.96 \pm 0.97 \mathrm{mg} / \mathrm{dl}$, respectively) compared to OP $(p<0.01)$.

\section{Calcium level and B-ALP activity in lysed osteogenic differentiated MSCs from MSCs-treated and MSCs- nontreated osteoporotic rats}

Calcium concentrations and B-ALP activity were measured in the lysed differentiated MSCs to indicate osteogenic differentiation of MSCs derived from different groups (Fig. 5). $\mathrm{Ca}$ concentration in the lysed differentiated cells of untouched control was $20.44 \pm 2.33 \mathrm{mg} /$ well and of the sham control group was $18.55 \pm 1.56 \mathrm{mg} /$ well. It was clear that $\mathrm{Ca}$ concentration in the lysed differentiated cells of sham control did not show any significant difference compared to the untouched control group. However, $\mathrm{Ca}$ concentrations in the lysed differentiated cells of OP at 4 weeks $(11.12 \pm 1.17 \mathrm{mg} /$ well $)$ and 8 weeks $(8.54 \pm 1.09 \mathrm{mg} /$ well $)$ were significantly decreased compared to the untouched control and sham control groups $(p<0.05)$. 


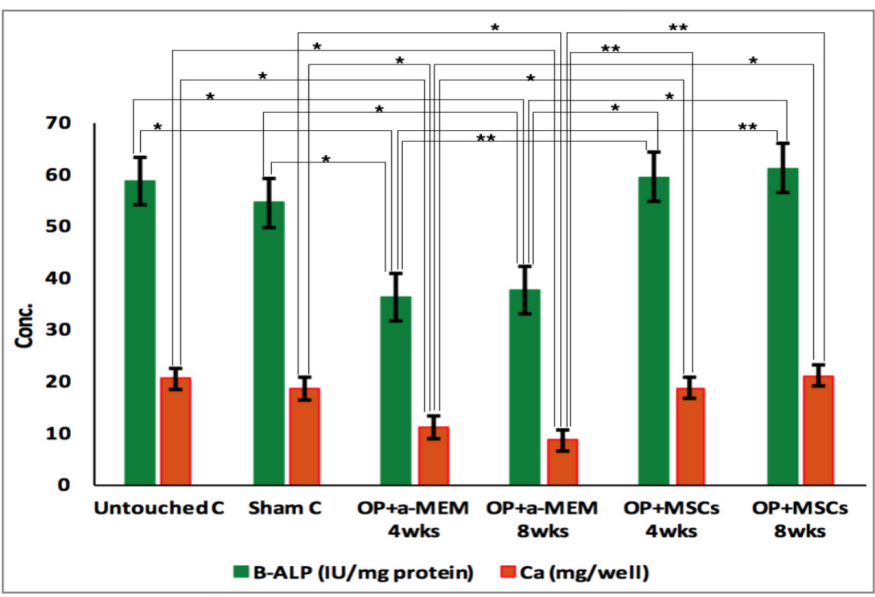

Figure 5. Calcium concentration and B-ALP activity in the lysed differentiated MSCs from the different groups of rats. Bars represent mean \pm SE of groups and marked by $*$ and $* *$ as significantly different at $p<0.05$ and $p<0.01$, respectively. C: control, OP: osteoporosis, $\alpha$-MEM: alpha-modified Eagle medium, and MSCs: mesenchymal stem cells.
The results revealed that MSCs treatment led to a significant elevation of $\mathrm{Ca}$ concentrations in the lysed differentiated cells as shown in the OP + MSCs group after either 4 weeks $(18.65 \pm$ $2.81 \mathrm{mg} /$ well $)$ or 8 weeks $(21.02 \pm 3.21 \mathrm{mg} /$ well $)$ compared to $\mathrm{OP}$ at 4 and 8 weeks $(p<0.01)$.

B-ALP activity in the lysed differentiated MSCs of sham control $(54.4 \pm 3.18 \mathrm{IU} / \mathrm{mg}$ protein) did not show any significant difference compared to the untouched control group $(58.6 \pm 4.11 \mathrm{IU} / \mathrm{mg}$ protein). The activities of B-ALP in the lysed differentiated MSCs of OP at 4 weeks $(36.11 \pm 3.75 \mathrm{IU} /$ $\mathrm{mg}$ protein) and 8 weeks $(37.55 \pm 2.65 \mathrm{IU} / \mathrm{mg}$ protein) show a significant decrease, as compared to the untouched control and sham control groups $(p<0.05)$. However, a significant elevation of B-ALP activity was noticed after MSCs treatment as shown in the lysed differentiated MSCs of the OP + MSCs group at 4 and 8 weeks $(59.45 \pm 4.54$ and $61.14 \pm 5.85 \mathrm{IU} / \mathrm{mg}$ protein, respectively) as compared to their corresponding OP groups $(p<0.01)$.
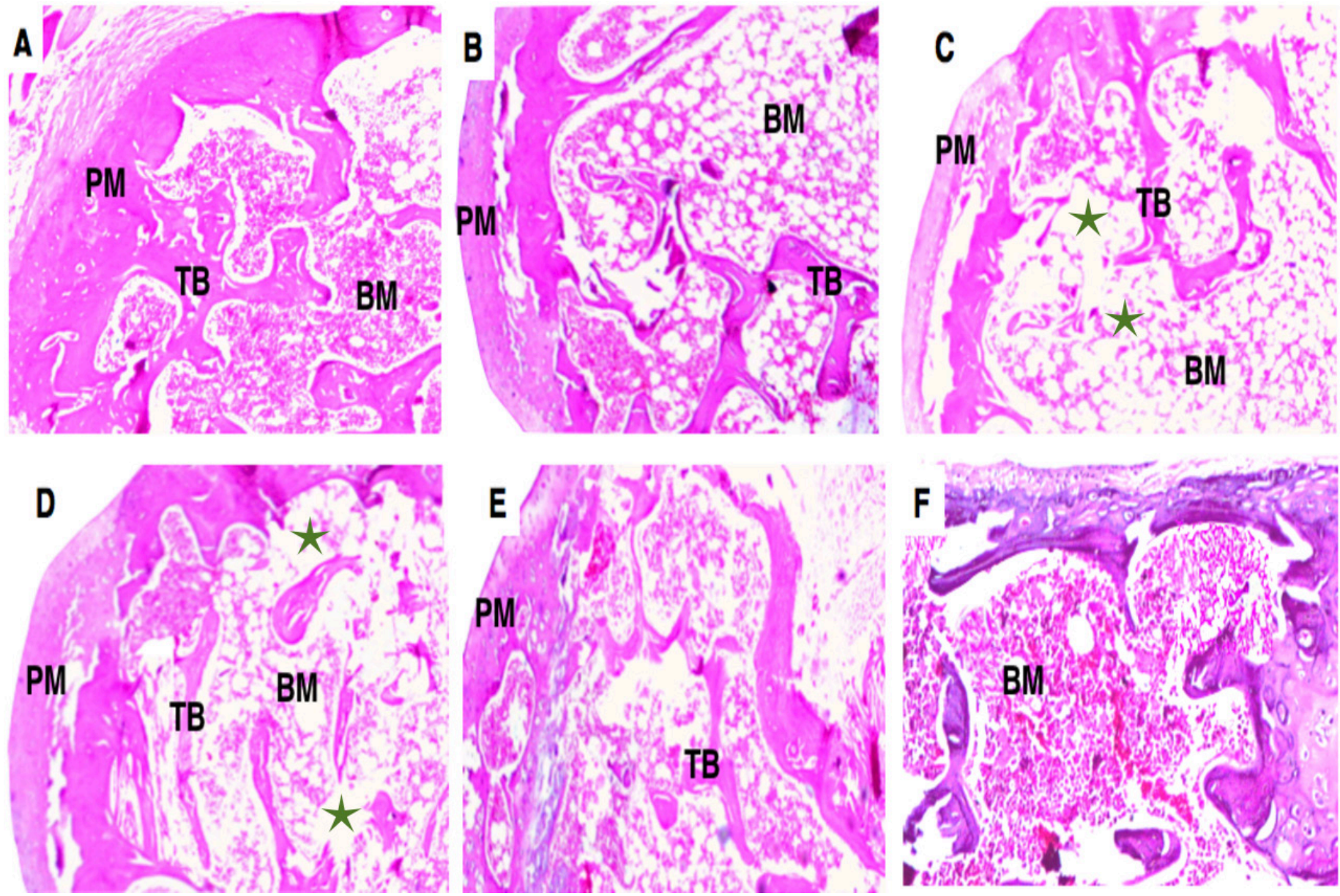

Figure 6. Histological architecture of soft regions of the left femur of different groups stained by hematoxylin-eosin. Untouched control (A) and sham control (B) showed uniform TB within abundant bone marrow. The PM is seen in the outermost layer of bone. Soft bone of OP group after 4 weeks (C) and 8 weeks (D) showed thin TB with wide intertrabecular spaces and disjointed bony ossicles interrupted by expanded fatty bone marrow spaces (green stars). On the other hand, cancellous bone of OP groups treated with MSCs showed normal TB with red bone marrow after 4 weeks (E) and 8 weeks (F). All micrographs were visualized by $\times 40$ magnification. OP: osteoporosis and MSCs: mesenchymal stem cells. 


\section{Bone histomorphometry from MSCs-treated and MSCs- nontreated osteoporotic rats}

The criteria for the histological sections of the left femoral diaphysis for the untouched and control groups stained by $H \& E$ presented normal red bone marrow and trabecular bone and periosteum (PM) with narrow intertrabecular spaces. The histopathological examination of the cancellous bone revealed continual thinning of the trabeculae (TB) in the OP after 4 or 8 weeks leading to widening of intertrabecular spaces in the head of the femur and intermittent bony ossicles interrupted with extended fatty bone marrow spaces. After MSCs treatment, cancellous bone TB appeared normal with red bone marrow (Fig. 6).

\section{In vitro osteoblastic differentiation from MSCs-treated and MSCs-nontreated osteoporotic rats}

In vitro osteoblastic differentiation of MSCs in response to culturing with osteogenic media was analyzed by Alizarin red staining (Fig. 7). The differentiation of MSCs showed highly red-stained dispersed calcified nodules of the untouched control (Fig. 7A) and sham control (Fig. 7B) groups in response to red
Alizarin. Photomicrograph of in vitro osteogenic differentiation of MSCs derived from OP group after 4 (Fig. 7C) and 8 (Fig. 7D) weeks showed very low scattered red calcified nodules in response to Alizarin red staining while treatment with MSCs in OP + MSC group expressed moderate to high scattered red calcified nodules after 4 (Fig. 7E) and 8 (Fig. 7F) weeks, respectively, after Alizarin red staining.

\section{DISCUSSION}

This approach determined the therapeutic potential of MSCs application in induced osteoporotic rats. The data obtained show that the transplantation of stem cells into osteoporotic rats provides a supplement for bone architecture and enhances osteoblastic differentiation, suggesting reverting OP. Bone formation is a biological process by which the old bone is replaced by a new one. The old bone was resorbed by osteoclasts while the new formation was caused by osteoblasts (Bellido, 2014). To date, it is not yet clear how osteogenesis or adipogenesis can be optimized and differentiated. MSCs are nonhematopoietic cells with the potentiality of self-renewal and multilineage differentiation and have been extensively used in the cellular therapeutic field
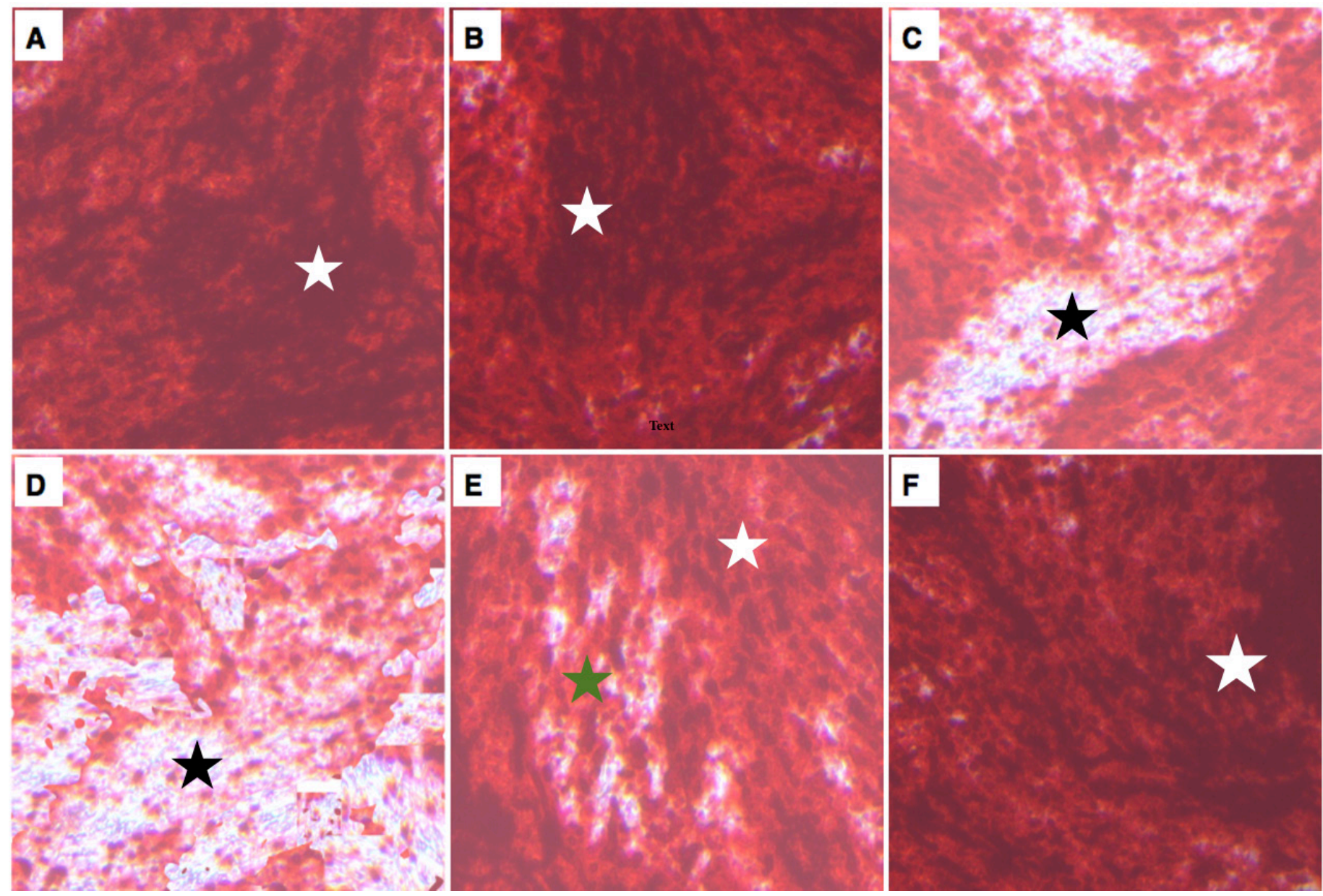

Figure 7. Photomicrograph of in vitro osteoblastic differentiation of MSCs derived from different groups of rats after Alizarin red staining. Untouched control (A) and sham control (B) showed highly scattered red calcified nodules (white star). Osteogenic differentiated cells of OP groups showed very low scattered red calcified nodules (black star) after 4 weeks (C) and 8 weeks (D) of induction. Osteogenic differentiated cells of OP + MSCs group expressed moderate (green star) to high (white star) scattered red calcified nodules after 4 weeks (E) and 8 weeks (F). All photomicrographs were visualized by $\times 40$ magnification. OP: osteoporosis and MSCs: mesenchymal stem cells. 
(Fortier and Travis, 2011). MSCs are the most interesting cells to date, because of their assorted functions. In addition, they have fascinating characteristics in tissues and organs regeneration, which suggest that MSCs are capable of directing toward injured tissue, to undergo differentiation and immunomodulation (Aguirre et al., 2010). In the current investigation, bone marrow MSCs were used for the treatment of osteoporotic rats, and before that, their morphology and phenotypic characteristics were examined and confirmed. Previous studies have shown that the OP was induced by ovariectomy (Kikuta et al., 2013), glucocorticoid supplementation (Aggarwal et al., 2012), or immune-deficient osteoporotic murine model (Aggarwal et al., 2014). In the present study, a model was chosen to induce OP in rats by ovariectomy and glucocorticoids injection for eight weeks. It was known that glucocorticoids have a dual effect, increase bone resorption decrease, and decrease bone formation (Khalifa et al., 2020). Ovariectomy removes the osteogenic effect of estrogen (Saito et al., 2018). Then stem cells were isolated for determination of its criteria and ability to relieve OP. In this investigation, data revealed that the MSCs viability \% and formazan OD derived from OP at 4 and 8 weeks were significantly decreased in comparison with the untouched and sham control groups. The calcium concentration and B-ALP activity were measured in the lysed differentiated MSCs to indicate osteogenic differentiation of MSCs derived from different groups. It was clear that calcium concentration and B-ALP activity in the lysed differentiated cells of sham control did not show any significant difference when compared to the untouched control group. However, calcium concentration and B-ALP activity in the lysed differentiated cells of OP at 4 and 8 weeks were significantly decreased as compared to the untouched and sham control groups. These results corroborate the findings on the OP rat model outlined by Khalifa et al. (2020).

In this investigation, the concentrations of $\mathrm{OCN}, \mathrm{Ca}$, $\mathrm{P}$ and B-ALP in serum of OP rats were obtained. A significant decline was noticed in the serum concentrations of $\mathrm{OCN}, \mathrm{Ca}$, and B-ALP activity of OP at 4 and 8 weeks compared to the untouched control and sham control groups. However, serum $\mathrm{P}$ concentration was significantly higher in OP at 4 and 8 weeks after induction compared to the untouched control and sham control groups. These results agree with the research conducted previously by (Khalifa et al., 2020). The histopathological image of the femur head displayed a constant reduction of the trabecular bone in the OP group after 4 or 8 weeks leading to broadening of spaces between TB and disjointed bony ossicles interrupted by expanded fatty bone marrow spaces. Photomicrograph of in vitro osteogenic differentiation of isolated and proliferated MSCs of untouched and sham control groups showing highly dispersed red to brown calcified nodules by Alizarin red staining. Photomicrograph of MSCs isolated from OP model after 4 and 8 weeks of induction and undergoing in vitro osteogenic differentiation showed very low dispersed red to brown calcified nodules by Alizarin red staining. The loss of stem cells from bone marrow and their osteoplastic progeny is assumed to be a prominent factor for reduction of bone mass and now stem cell therapies for OP were under consideration, but their success in clinical applications is yet to be established. Due to the plenty of MSCs from bone marrow, it can be considered as an ultimate cell-based therapy to treat bone disease like OP. Due to ethics concerning embryonic stem cell usage, laboratory attention has been focused on adult stem cells. However, MSCs combined two basic characteristics of self-renewal and multilineage differentiation. Bei et al. (2018) showed that MSCs have the ability to alleviate OP locally and systemic, through direct and indirect mechanisms. Systematic manipulation was preferred as in the present study to avoid the traumatic issue by local injection. The mechanism by which MSCs alleviate OP might be the engraftment to local osteoporotic lesion or direct anabolism of osteogenic differentiation or secretion of tropic factors for osteoblast (Sui et al., 2016).

It was noticed in the present study that cell viability $\%$ and formazan OD of MSCs derived from OP + MSCs after 4 and 8 weeks after treatment were significantly higher than that of OP at 4 and 8 weeks. The results revealed also that $\mathrm{Ca}$ concentration and B-ALP activity in the lysed differentiated cells of OP + MSCs group at 4 weeks after treatment were significantly elevated compared to OP at 4 and 8 weeks. After treatment with $\mathrm{MSCs}$, the results revealed that $\mathrm{OCN}, \mathrm{Ca}$, and $\mathrm{OCN}$ concentration and B-ALP activity in the serum of OP + MSCs rats at 4 weeks after treatment were elevated compared to OP at 4 and 8 weeks. On the other hand, OP + MSCs showed a significant decrease in serum $\mathrm{P}$ concentration at either 4 or 8 weeks after MSCs treatment compared to OP at 4 and 8 weeks. The histological image showed that, after MSCs treatment, cancellous bone TB appeared normal with red bone marrow. This data indicated a significant elevation of bone formation rate with the restoration of estrogen deficiencyinduced trabecular and cortical bone loss due to ovariectomy as previously reported that bone histomorphometry analysis revealed a reduction in the number and width of trabecular bone of the tibia in OP rats (Yao et al., 2013).

MSCs have the ability to alleviate OP through local and systemic or direct and indirect mechanisms (Sui et al., 2017). In the present study, due to traumatic issues by local injection, the mechanism by which MSCs alleviate OP might be the engraftment to local osteoporotic lesion or direct anabolism of osteogenic differentiation or secretion of trophic factors for osteoblast (Sui et al., 2016). Photomicrograph of in vitro osteogenic differentiation of MSCs of OP after 4 and 8 weeks showed very low dispersed red to brown nodules by Alizarin red staining while treatment with MSCs of OP expressed moderate to high scattered red calcified nodules after 4 and 8 weeks, respectively, in response to Alizarin red staining. This is consistent with the previously reported in vitro findings (Shen et al., 2018; Sui et al., 2018) which attributed the relief to immunosuppression/anti-inflammation and resident stem cell recovery.

\section{CONCLUSION}

Based on the present findings, it is concluded that the transplantation of MSCs into osteoporotic rats augments bone architecture and enhances osteoblastic differentiation and function, resulting in reverting OP because these cells provide the required complementary effect to the bone. This present study provides evidence for the nonimmunogenic therapy of MSCs for the treatment of osteoporotic murine models. This work presents a unique method as a future application for the treatment of human OP. Pharmacotherapy can be prohibitive in cost, taking a long time to be effective, and lead to adverse reactions. Bisphosphonate 
therapy may lead to extensive suppression of bone development. Therefore, the combination of MSCs with bisphosphonate is put in perspective for control of OP, which needs to address many of the challenges that exist in this application in the future study.

\section{AUTHORS' CONTRIBUTION}

ASMA, AAZ, and FAMA contributed to the conception and design of the study. ASMA, WA, ZR, and FAA contributed to the acquisition of data. ASMA, WA, ZR, FAA, and FAMA contributed to the analysis and interpretation of data. ASMA, AAZ, and FAMA prepared the final manuscript for submission. All authors read, revised, and approved the final version of the article for submission.

\section{ACKNOWLEDGMENTS}

The authors gratefully acknowledge Qassim University represented by the Deanship of Scientific Research for the material support to conduct this research through grant number 3739-cavm-2018-1-14-S.

\section{CONFLICT OF INTEREST}

The authors have no conflicting financial interest.

\section{FUNDING}

This study is financially supported by the Deanship of Scientific Research, Qassim University, KSA, project no. 3739-cavm-2018-1-14-S.

\section{PUBLISHER'S NOTE}

This journal remains neutral with regard to jurisdictional claims in published institutional affiliation.

\section{REFERENCES}

Abo-Aziza FAM, Zaki AA, Amer AS, Lotfy RA Dihydrotestosterone and 17-estradiol enhancement of in vitro osteogenic differentiation of castrated male rat bone marrow mesenchymal stem cells (rBMMSCs). Int J Hematol Oncol Stem Cell Res, 2019a; 13(4):208-19.

Abo-Aziza FAM, Zaki AA, El-Maaty AMA. Bone marrowderived mesenchymal stem cell (BM-MSC): a tool of cell therapy in hydatid experimentally infected rats. Cell Reg, 2019b; 8(2):58-71.

Aggarwal R, Lu J, Kanji S, Joseph M, Das M, Noble GJ, McMichael BK, Agarwal S, Hart RT, Sun Z, Lee BS. Human umbilical cord blood-derived CD34+ cells reverse osteoporosis in NOD/SCID mice by altering osteoblastic and osteoclastic activities. PLoS One, 2012; 7(6):e39365.

Aggarwal R, Pompili VJ, Das H. Generation of osteoporosis in immune-compromised mice for stem cell therapy. Anim Mod Stem Cell Ther, 2014; 1213:209-14.

Aguirre A, Planell J, Engel E. Dynamics of bone marrow-derived endothelial progenitor cell/mesenchymal stem cell interaction in co-culture and its implications in angiogenesis. Biochem Biophys Res Commun, 2010 400(2):284-91.

Alabdulmonem W, Alhomaidan HT, Rasheed Z, Madar IH, Alasmael N, Alkhatib S, Ssadh HA. CD74 a potential therapeutic target for breast cancer therapy: interferon gamma up-regulates its expression in CAMA-1 and MDA-MB-231 cancer cells. Int J Cancer Res, 2018; 14(2):58-69.

Almeida AL, Freitas GP, Lopes HB, Gimenes R, Siessere S, Sousa LG, Beloti MM, Rosa AL. Effect of stem cells combined with a polymer/ceramic membrane on osteoporotic bone repair. Brazilian Oral Res, 2019; 33:e079.
Bei J, Zhang X, Wu J, Hu Z, Xu B, Lin S, Cui L, Wu T, Zou L. Ginsenoside Rb1 does not halt osteoporotic bone loss in ovariectomized rats. PLoS One, 2018; 13:e0202885.

Bellido T. Osteocyte-driven bone remodeling. Calc Tissue Int, 2014; 94(1):25-34.

Chen J, Li K, Pang Q, Yang C, Zhang H, Wu F, Cao H, Liu $\mathrm{H}$, Wan Y, Xia W, Wang J. Identification of suitable reference gene and biomarkers of serum miRNAs for osteoporosis. Sci Rep, 2016; 8(6):36347.

Chen Y, Cheung KM, Kung HF, Leong JC, Lu WW, Luk KD. In vivo new bone formation by direct transfer of adenoviral-mediated bone morphogenetic protein-4 gene. Biochem Biophys Res Commun, 2002; 298(1):121-7.

Choi YS, Park SN, Suh H. Adipose tissue engineering using mesenchymal stem cells attached to injectable PLGA spheres. Biomaterials, 2005; 26(29):5855-63.

Cosman F, de Beur SJ, LeBoff MS, Lewiecki EM, Tanner B, Randall S, Lindsay R. Clinician's guide to prevention and treatment of osteoporosis. Osteopor Int, 2014; 25(10):2359-81.

Drake MT, Clarke BL, Lewiecki EM. The pathophysiology and treatment of osteoporosis. Clin Ther, 2015; 37(8):1837-50.

Eça LP, Ramalho RB, Oliveira IS, Gomes PO, Pontes P, Ferreira AT, Mazzetti MP. Comparative study of technique to obtain stem cells from bone marrow collection between the iliac crest and the femoral epiphysis in rabbits. Acta Cirúrg Brasil, 2009; 24(5):400-4.

Fortier LA, Travis AJ. Stem cells in veterinary medicine. Stem Cell Res Ther, 2011;2(1):9.

Huang GJ, Gronthos S, Shi S. Mesenchymal stem cells derived from dental tissues vs. those from other sources: their biology and role in regenerative medicine. J Dent Res, 2009; 88(9):792-806.

Khalifa AK, Diab SA, Hashem GM, Alalkamy EF, Yacoub MF. New promising avenue for the simvastatin combination with residronate, strontium ranelate and raloxifene in experimentally-induced osteoporosis. Egypt Rheumato, 2020; 42(1):63-9.

Kikuta S, Tanaka N, Kazama T, Kazama M, Kano K, Ryu J, Tokuhashi Y, Matsumoto T. Osteogenic effects of dedifferentiated fat cell transplantation in rabbit models of bone defect and ovariectomy-induced osteoporosis. Tiss Eng Part A, 2013; 19(15-16):1792-802.

Lee SH, Cha SH, Kim CL, Lillehoj HS, Song JY, Lee KW. Enhanced adipogenic differentiation of bovine bone marrow-derived mesenchymal stem cells. J App Anim Res, 2015; 43(1):15-21.

Littman N, Abo A. Proceedings: using stem cell therapies to reestablish osteogenic capability for bone regeneration. Stem Cells Trans Med, 2015; 4(11):1247-50.

Makras P, Delaroudis S, Anastasilakis AD. Novel therapies for osteoporosis. Metabolism, 2015; 64(10):1199-214.

Miyamoto T. Mechanism underlying post-menopausa osteoporosis: HIF $1 \alpha$ is required for osteoclast activation by estrogen deficiency. Keio J Med, 2015; 64(3):44-7.

Quan R, Zheng X, Ni Y, Xie S, Li C. Culture and characterization of rat hair follicle stem cells. Cytotechnology, 2016; 68(4):621-8.

Rasheed Z, Akhtar N, Khan A, Khan KA, Haqqi TM. Butrin, isobutrin, and butein from medicinal plant Butea monosperma selectively inhibit nuclear factor-kappaB in activated human mast cells: suppression of tumor necrosis factor-alpha, interleukin (IL)-6, and IL-8. J Pharmacol Exp Ther, 2010; 333(2):354-63.

Rousselle AV, Heymann D. Osteoclastic acidification pathways during bone resorption. Bone, 2002; 30(4):533-40.

Salasznyk RM, Klees RF, Hughlock MK, Plopper GE. ERK signaling pathways regulate the osteogenic differentiation of human mesenchymal stem cells on collagen I and vitronectin. Cell Commun Adhes, 2004; 11(5-6):137-53.

Sanghani-Kerai A, Coathup M, Samazideh S, Kalia P, Silvio LD, Idowu B, Blunn G. Osteoporosis and ageing affects the migration of stem cells and this is ameliorated by transfection with CXCR4. Bone Joint Res, 2017; 6(6):358-65. 
Saito A, Nagaishi K, Iba K, Mizue Y, Chikenji T, Otani M, Nakano M, Oyama K, Yamashita T, Fujimiya M. Umbilical cord extracts improve osteoporotic abnormalities of bone marrow-derived mesenchymal stem cells and promote their therapeutic effects on ovariectomised rats. Sci Rep, 2018; 8:1-16.

Shen M, Wu R, Jin R, Pan J, Guo F, Li Z, Lin X, Xu S. Injection of synthetic mesenchymal stem cell mitigates osteoporosis in rats after ovariectomy. J Cell Mol Med, 2018; 22(8):3751-7.

Ssadh HA, Abdulmonem WA, Rasheed Z, Madar IH, Alhoderi J, Eldeen SKN, Alradhwan A, Alasmael N, Alkhamiss A, Fernández N. Knockdown of CD-74 in the proliferative and apoptotic activity of breast cancer cells. Open Access Maced J Med Sci, 2019; 7(19):3169-76.

Sui B, Hu C, Zhang X, Zhao P, He T, Zhou C, Qiu X, Chen $\mathrm{N}$, Zhao X, Jin Y. Allogeneic mesenchymal stem cell therapy promotes osteoblastogenesis and prevents glucocorticoid-induced osteoporosis. Stem Cells Trans Med, 2016; 5(9):1238-46.

Sui BD, Chen J, Zhang XY, He T, Zhao P, Zheng CX, Li M, Hu $\mathrm{CH}$, Jin Y. Gender-independent efficacy of mesenchymal stem cell therapy in sex hormone-deficient bone loss via immunosuppression and resident stem cell recovery. Exp Mol Med, 2018; 50(12):1-4.

Sui BD, Hu CH, Zheng CX, Shuai Y, He XN, Gao PP, Zhao P, Li M, Zhang XY, He T, Xuan K. Recipient glycemic micro-environments govern therapeutic effects of mesenchymal stem cell infusion on osteopenia. Theranostics, 2017; 7(5):1225.

Sun S, Sun L, Kang Y, Tang L, Qin YX, Ta D. Therapeutic effects of low-intensity pulsed ultrasound on osteoporosis in ovariectomized rats: intensity-dependent study. Ultrasound Med Biol, 2020; 46(1):108-21.
Thomsen JS, Christensen LL, Vegger JB, Nyengaard JR, Brüel A. Loss of bone strength is dependent on skeletal site in disuse osteoporosis in rats. Calc Tissue Int, 2012; 90(4):294-306.

Wronski T, Lowry P, Walsh C, Ignaszewski L. Skeletal alterations in ovariectomized rats. Calc Tiss Int, 1985; 37(3):324-8.

Yao W, Guan M, Jia J, Dai W, Lay YA, Amugongo S, Liu R, Olivos D, Saunders M, Lam KS, Nolta J. Reversing bone loss by directing mesenchymal stem cells to bone. Stem Cells, 2013; 31(9):2003-14.

Zaki AA, Tariq I. Almundarij TI, Abo-Aziza FAM. Comparative characterization and osteogenic/adipogenic differentiation of mesenchymal stem cells derived from male rat hair follicles and bone marrow. Cell Reg, 2020; 9:13.

\section{How to cite this article:}

Aljohani ASM, Zaki AK, Abdulmonem WA, Rasheed Z, Alhumaydhi FA, Abo-Aziza FAM. Therapeutic approach for osteoporotic animal model by bone marrow mesenchymal stem cells. J Appl Pharm Sci, 2022; 12(01):149-158. 